\title{
Early rebrightenings of X-ray afterglows from ring-shaped GRB jets
}

\author{
M. $\mathrm{Xu}^{1,2}$ and Y. F. Huang ${ }^{1,2}$ \\ 1 Department of Astronomy, Nanjing University, Nanjing 210093, PR China \\ e-mail: hyf@nju.edu.cn \\ ${ }^{2}$ Key Laboratory of Modern Astronomy and Astrophysics (Nanjing University), Ministry of Education, PR China
}

Received 4 March 2010 / Accepted 6 August 2010

\begin{abstract}
Aims. Early rebrightenings at a post-burst time of $10^{2}-10^{4} \mathrm{~s}$ have been observed in the afterglows of some gamma-ray bursts (GRBs). Unlike X-ray flares, these rebrightenings usually last for a comparatively long period. The continuous energy injection mechanism can usually only produce a plateau in the afterglow light curve, but not a rebrightening. Also, a sudden energy injection can induce a rebrightening, but the rebrightening is slightly too rapid.

Methods. We argue that the early rebrightenings can be produced by a ring-shaped jet, for which we here present a model. In this scenario, the GRB outflow is not a full cone, but a centrally hollowed ring. Assuming that the line of sight is on the central symmetry axis of the hollow cone, we calculate the dynamical evolution of the outflows and the multiband afterglow light curves.

Results. We find that the early rebrightenings observed in the afterglows of some GRBs (i.e. approximately $1 \%$ of $S w i f t$ events), such as GRB 051016B, 060109, 070103, and 070208, can be reproduced with this model.

Conclusions. We suggest that these long-lasting early rebrightenings in GRB afterglows are the result of ring-shaped jets.
\end{abstract}

Key words. gamma-ray burst: general - ISM: jets and outflows

\section{Introduction}

Gamma-ray bursts (GRBs) are powerful explosive events in the Universe. The standard fireball model suggests that the prompt emission is a result of internal shocks and that the afterglow results from external shocks. Since the launch of the Swift satellite (Gehrels et al. 2004), enormous improvements have been achieved in understanding the nature of GRBs (for recent reviews, see: Zhang 2007; Gehrels et al. 2009). Generally, some interesting components have been identified in the X-ray afterglow light curves of GRBs, i.e., the steep decay phase, the shallow decay phase, the normal decay phase, the post jet-break phase, and X-ray flares.

Rebrightening behavior is also a very interesting feature among a few GRBs. Unlike X-ray flares, which are usually characterized by a rapid rise and fall of the flux (with the mean ratio of width-to-peak time $\langle\Delta t / t\rangle=0.13 \pm 0.1$, Chincarini et al. 2007), these rebrightenings are generally very gentle, with $\Delta t / t>1$. Rebrightenings can occur either in the early afterglow stage $\left(t \leq 10^{4} \mathrm{~s}\right)$ or in the late afterglow stage $\left(t \gg 10^{4} \mathrm{~s}\right)$. Rebrightening in the late stage was first discovered in the X-ray and optical afterglow of GRB 970508, at a post-burst time of about $t \sim 2$ days (Piro et al. 1998). More examples of late rebrightenings (typically occuring at $t \geq 1-2$ day) can be found in GRBs 030329 (Lipkin et al. 2004), 031203 (Ramirez-Ruiz et al. 2005), 050408 (de Ugarte Postigo et al. 2007), and 081028 (Margutti et al. 2009). Interpretations for these late rebrightenings include an off-axis jet model and microphysics variation mechanism (Kong et al. 2010).

In this study, we will mainly concentrate on the early rebrightenings that typically occur at a post-burst time of $t \leq$ $10^{4} \mathrm{~s}$. Examples of these early rebrightenings can be found in GRBs 051016B, 060109, 070103, 070208 etc. Most GRBs, like GRB 061007 (Kocevski \& Butler 2008), do not exhibit early rebrightenings. Among more than 500 Swift GRBs (Gehrels et al. 2009) we note that only less than $1 \%$ events show early Xray rebrightenings. The energy-injection mechanism is a natural explanation for rebrightenings, but it is unlikely to take effect here. For example, the continuous energy-injection mechanism is usually more likely to produce a plateau-like structure in the afterglow light curve, but not an obvious rebrightening (Dai \& Lu 1998; Zhang \& Mészáros 2001; Yu \& Huang 2007). Also, although a sudden energy-injection can give birth to a rebrightening, the rebrightening is generally slightly too rapid (Huang et al. 2006) compared with the early rebrightenings considered here.

We suggest that these early rebrightenings may be produced by ring-shaped GRB jets. A ring-shaped jet is not a full cone, but a centrally hollowed ring (Granot 2005; Fargion \& Grossi 2006; $\mathrm{Xu}$ et al. 2008). In this paper, we numerically investigate the afterglow features of these outflows and use this mechanism to explain several observed afterglows. The structure of our paper is as follows. The geometry of ring-shaped jets is sketched in Sect. 2. The dynamical evolution and afterglow of ring-shaped jets are studied in Sect. 3 together with a detailed comparison with a few observations. Finally, Sect. 4 presents our discussion and conclusion.

\section{Ring-shaped jet}

A ring-shaped jet can be produced by the central engine during the prompt GRB phase. The most natural mechanism may be via precessing (Fargion \& Grossi 2006; Zou \& Dai 2006). In almost all GRB progenitor models, the central engine is a rapidly rotating compact object, such as a black hole or a neutron star. The outflows accounting for the GRB are usually launched along the rotating axis or the magnetic axis. If the nozzle of 
the central engine is precessing while ejecting the outflow, then a ring-shaped jet will naturally be produced. As an example, if GRBs are associated with the kick of neutron stars (Huang et al. 2003), then the production of ring-shaped jets will be quite likely. Alternatively, the apparent ring-shaped topology of the outflow can also arise from a structured jet as suggested by van Putten \& Levinson (2003) and van Putten \& Gupta (2009). This mechanism can explain the correlation between the observed luminosity and variability in the light curves of long GRBs reported by Reichert et al. (2001).

A detailed description of the model for ring-shaped jets has been presented in Xu et al. (2008) and we use the same notations here. The half-opening angle of the inner edge of the ring is denoted as $\theta_{c}$, and the width of the ring is $\Delta \theta$. Both the inner and outer edges may expand laterally, so the inner edge will converge at the central axis and the ring-shaped jet will finally become a conical jet after a period of time. In our calculations we assume for simplicity that the line of sight is on the symmetry axis of the centrally hollowed cone. Therefore, this scenario is more or less similar to that of a normal off-axis jet at early stages, i.e. the line of sight is not on the emitting outflow. A rebrightening is then expected to be present in the early afterglow phase, thanks to the deceleration and lateral expansion of the ring-shaped jet.

Because our line of sight is initially "off-axis", a natural problem is whether the ring-shaped jet could produce the intensive $\gamma$-ray radiation in the prompt GRB phase, similar to the problem confronted by a normal off-axis jet model. Below we show that the ring-shaped jet model has more advantages compared to the off-axis jet model where this problem is concerned. The key point is that all material on a ring can contribute equally to the flux on the central axis.

Assuming that the bulk Lorentz factor of a highly relativistic point-like jet is $\gamma \gg 1$, and that it is viewed from an angle $\Theta$ relative to its motion, then the observed flux will be amplified by a factor of $\sim D^{3}$, owing to the effect of relativistic beaming and Doppler boosting. Here the Doppler factor is $D=[\gamma(1-$ $\beta \cos \Theta)]^{-1}$, with $\beta=\sqrt{1-1 / \gamma^{2}}$. For an extended outflow such as is considered in our framework, we need to integrate over the whole surface of the ring-shaped jet to get the exact factor of amplification for the prompt GRB emission.

We first consider a normal conical jet with a full opening angle of $\Delta \theta$ and a viewing angle of $\theta_{\mathrm{c}}+\frac{1}{2} \Delta \theta$ (similar to the viewing angle of our ring-shaped jet scenario). The observed emission will be amplified by a factor of $f_{\mathrm{j}}=\int_{\theta_{\mathrm{c}}}^{\theta_{\mathrm{c}}+\Delta \theta} D^{3} \phi(\Theta) \mathrm{d} \Theta$ relative to that in the jet rest frame, where $\phi(\Theta)$ is the toroidal angle given by

$\phi(\Theta)=2 \arccos \left[\frac{\cos (\Delta \theta / 2)-\cos \left(\theta_{\mathrm{c}}+\Delta \theta / 2\right) \cos \Theta}{\sin \left(\theta_{\mathrm{c}}+\Delta \theta / 2\right) \sin \Theta}\right]$.

For simplicity we define the corresponding amplification factor as $f_{\mathrm{o}}$ for a normal conical GRB jet with a full opening angle of $\Delta \theta$ but with the viewing angle of $\sim 1 / \gamma$. Actually, $f_{\mathrm{o}}$ is a measure of the amplification factor for an on-axis observer.

Now we return to the ring-shaped jet considered in our framework. The amplification factor for an observer on the central symmetry axis is $f_{\mathrm{r}}=\int_{\theta_{\mathrm{c}}}^{\theta_{\mathrm{c}}+\Delta \theta} D^{3} \phi(\Theta) \mathrm{d} \Theta$, where $\phi(\Theta)=2 \pi$. To get a direct impression on this matter, let us simply take $\gamma=50, \theta_{\mathrm{c}}=0.04, \Delta \theta=0.04$ as an example, and calculate the final amplification factors. In this example, the viewing angle, i.e. the angle between the jet axis and the line of sight, equals $3 / \gamma$. We can easily find that $f_{\mathrm{o} / \mathrm{r}} \equiv f_{\mathrm{o}} / f_{\mathrm{r}} \approx 68.5$. It means that the observed flux of our ring-shaped jet is only tens of times less than that of an on-axis jet in the prompt GRB phase. At the same time, we can also find that $f_{\mathrm{r} / \mathrm{j}} \equiv f_{\mathrm{r}} / f_{\mathrm{j}} \approx 12.4$. It tells us that the observed flux of our ring-shaped jet is more than 12 times higher than that of an off-axis conical jet. Consequently, the ring-shaped jet model is superior to the normal off-axis conical jet model when considering the production of the intensive $\gamma$-ray radiation in the prompt GRB phase.

\section{Numerical results}

The overall dynamical evolution of a ring-shaped jet has been studied in detail by Xu et al. (2008). Generally, if the line of sight is on the central symmetry axis of the hollow cone, a rebrightening phase should present in the early afterglow light curve. Here we simply outline for completeness a few important parameters of the calculation. First, the dynamical equations should be appropriate for both the ultra-relativistic and non-relativistic stages. Recently, van Eerten et al. (2010) developed an accurate and also correspondingly complex code for the dynamical evolution of GRB afterglows. Here, we will use the simple and convenient equations proposed by Huang et al. (1999). Secondly, for a ring-shaped jet, both the inner and outer edges may expand laterally. When the inner edge converges at the central symmetry axis, the ring-shaped jet will become a normal conical jet, and then we only need to consider the sideways expansion of the outer edge. We suppose that the lateral expansion is at the comoving sound speed $\left(c_{\mathrm{s}}\right)$ approximately given by

$c_{\mathrm{s}}^{2}=\hat{\gamma}(\hat{\gamma}-1)(\gamma-1) \frac{1}{1+\hat{\gamma}(\gamma-1)} c^{2}$,

where $\hat{\gamma} \approx(4 \gamma+1) /(3 \gamma)$ is the adiabatic index (Dai et al. 1999). We have $\hat{\gamma} \sim 4 / 3$ and $c_{\mathrm{s}}=c / \sqrt{3}$ in ultrarelativistic limit, and $\hat{\gamma} \sim 5 / 3$ and $c_{\mathrm{s}}=\sqrt{5 / 9} \beta c$ in nonrelativistic limit. The effect of $c_{\mathrm{S}}$ on the light curve of a ring-shaped jet has been discussed by $\mathrm{Xu}$ et al. (2008) in detail. Generally speaking, lateral expansion tends to make the light curve steeper and leads to an earlier jet break. Thirdly, to calculate the afterglow flux, we integrate the emission over the equal arrival time surface defined by

$$
\int \frac{1-\beta \cos \Theta}{\beta c} \mathrm{~d} R \equiv t
$$

within the jet boundaries (Huang et al. 2000).

In this section, we will calculate the overall dynamical evolution and multiband afterglow of ring-shaped jets, and compare the numerical results with the observations of some Swift GRBs with early rebrightenings, such as GRBs 051016B, 060109, 070103, and 070208. The parameters used in our calculations are shown in Table 1 . Note that in this table, $E_{\mathrm{K} \text {,iso }}$ is the isotropicequivalent kinetic energy of the outflow, $n$ is the number density of the circum-burst medium, $\epsilon_{\mathrm{e}}$ and $\epsilon_{\mathrm{B}}$ are the energy fractions of electrons and magnetic field respectively, and $p$ is the power-law index of the energy distribution function of electrons. $1 \sigma$ error ranges are given for all the parameters. In our calculations, we assume a standard cosmology with $\Omega_{\mathrm{M}}=0.27, \Omega_{\Lambda}=0.73$, and with the Hubble constant of $H_{0}=71 \mathrm{~km} \mathrm{~s}^{-1} \mathrm{Mpc}^{-3}$.

\subsection{GRB $051016 B$}

GRB 051016B was triggered and located as a soft burst by SwiftBAT at 18:28:09 UT on 2005 October 16 (Parsons et al. 2005). The light curve of its prompt emission is shown in Fig. 1a. Its duration is $T_{90}=4.0 \pm 0.1 \mathrm{~s}$. The photon index in $15-150 \mathrm{keV}$ 
Table 1. Parameters of the four GRBs.

\begin{tabular}{lcccc}
\hline \hline GRB name & GRB 051016B & GRB 060109 & GRB 070103 & GRB 070208 \\
\hline$z$ & 0.9364 & 1 & 1 & 1.165 \\
$E_{\gamma \text {,iso }}\left(10^{51} \mathrm{erg}\right)$ & $0.76 \pm 0.09$ & $3.37 \pm 0.53$ & $1.79 \pm 0.26$ & $3.32 \pm 0.77$ \\
$E_{\mathrm{K}, \text { iso }}\left(10^{52} \mathrm{erg}\right)$ & $1.3 \pm 0.01$ & $6.0 \pm 0.04$ & $4.0 \pm 0.12$ & $8.0 \pm 0.03$ \\
$\theta_{\mathrm{c}}\left(10^{-2} \mathrm{rad}\right)$ & $1.0 \pm 0.013$ & $1.8 \pm 0.055$ & $1.0 \pm 0.075$ & $0.9 \pm 0.012$ \\
$\Delta \theta\left(10^{-2} \mathrm{rad}\right)$ & $7.0 \pm 0.2$ & $2.4 \pm 0.027$ & $8.0 \pm 0.44$ & $2.2 \pm 0.014$ \\
$\gamma$ & $180 \pm 6.0$ & $90 \pm 1.6$ & $120 \pm 6.4$ & $150 \pm 1.5$ \\
$n\left(10^{-2} \mathrm{~cm}^{-3}\right)$ & $0.5 \pm 0.007$ & $8.0 \pm 0.15$ & $10.0 \pm 0.95$ & $10.0 \pm 0.1$ \\
$\epsilon_{\mathrm{e}}$ & $0.1 \pm 0.0008$ & $0.1 \pm 0.0007$ & $0.1 \pm 0.0038$ & $0.1 \pm 0.0005$ \\
$\epsilon_{\mathrm{B}}\left(10^{-4}\right)$ & $5.0 \pm 0.065$ & $1.0 \pm 0.01$ & $1.0 \pm 0.07$ & $1.0 \pm 0.006$ \\
$p$ & $2.05 \pm 0.00003$ & $2.2 \pm 0.0085$ & $2.75 \pm 0.011$ & $2.03 \pm 0.0002$ \\
$f_{\mathrm{r} / \mathrm{j}}$ & $5.8 \pm 0.1$ & $10.4 \pm 0.3$ & $5.3 \pm 0.3$ & $7.7 \pm 0.2$ \\
$f_{\mathrm{o} / \mathrm{r}}$ & $228.7 \pm 45.0$ & $48.5 \pm 11.7$ & $52.2 \pm 13.3$ & $61.0 \pm 7.0$ \\
\hline
\end{tabular}

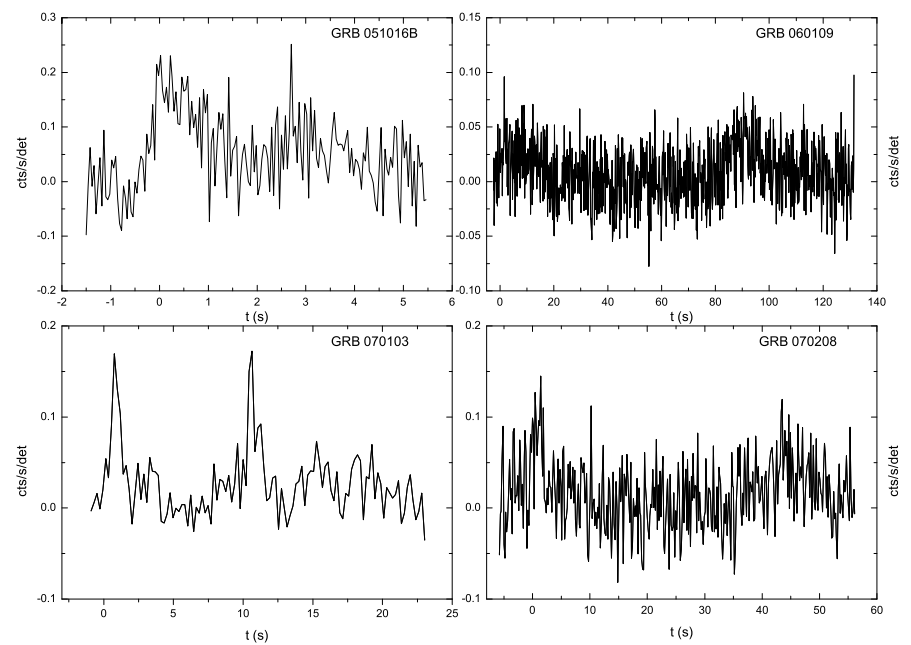

Fig. 1. Prompt $\gamma$-ray light curves of GRBs 051016B, 060109, 070103, and 070208 as observed by Swift-BAT (15-350 keV). The data are taken from the Swift website ${ }^{1}$ (Butler \& Kocevski 2007; Butler et al. 2007).

is $2.38 \pm 0.23$, and the fluence is $(1.7 \pm 0.2) \times 10^{-7} \mathrm{erg} \mathrm{cm}^{-2}$ (Barbier et al. 2005). Lying at a redshift of $z=0.9364$ (Soderberg et al 2005), the isotropic-equivalent $\gamma$-ray energy release is $E_{\gamma, \text { iso }} \sim(7.6 \pm 0.9) \times 10^{50} \mathrm{erg}$. The light curve of the early $\mathrm{X}$-ray afterglow shows a rising phase beginning at about $200 \mathrm{~s}$, and the rebrightening lasts for almost one thousand seconds.

Using the ring-shaped jet model, we fitted the observed $\mathrm{X}$-ray and optical afterglow light curves of GRB 051016B numerically. The result is presented in Fig. 2, and the parameter values are given in Table 1 . The observed X-ray afterglow data are relatively abundant. We see that our model can reproduce the rebrightening and the overall X-ray afterglow light curve satisfactorily. Note that in the very early stage $(t \leq 200 \mathrm{~s})$, the afterglow may still be in the steep decay phase and the flux should be dominant by the contribution from the prompt tail emission. We thus excluded these early data points from our modeling. In Fig. 2, the observed $R$-band optical afterglow light curve is built up only by two data points, but they agree well with our prediction. From Table 1, we see that the ratio of $f_{\mathrm{r} / \mathrm{j}}$ is about 5.8. It means that in the prompt GRB phase the $\gamma$-ray flux of our model exceeds about six times than that of a corresponding offaxis conical jet. Consequently, the ring-shaped jet model is better than the off-axis conical jet model when considering the production of the intensive $\gamma$-ray radiation in the main burst phase.

\footnotetext{
${ }^{1}$ http://astro. berkeley.edu/ nat/swift/
}

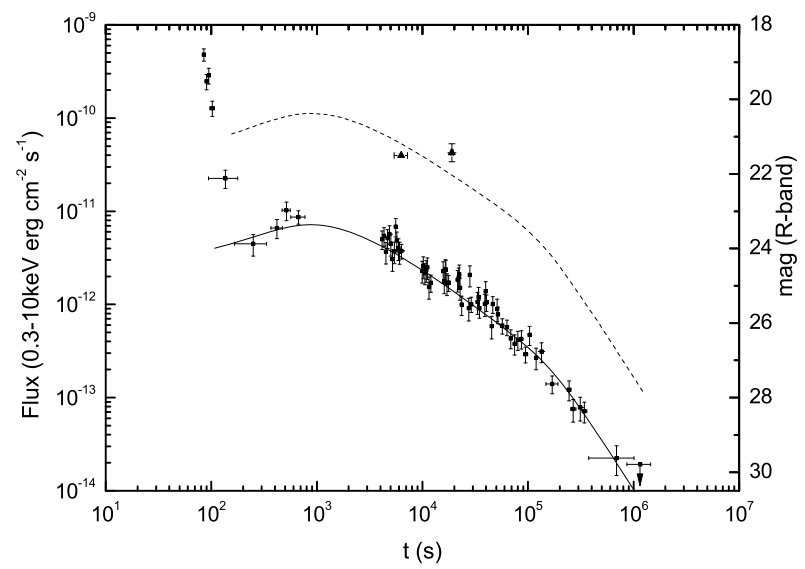

Fig. 2. Our best fit to the X-ray (solid line) and $R$-band optical (dashed line) afterglow light curves of GRB $051016 \mathrm{~B}$ by using the ring-shaped jet model. The square data points are observed X-ray afterglow by Swift-XRT (see the Swift website) and the triangle data points are observed $R$-band afterglow (Chen et al. 2005; Sharapov et al. 2005).

\subsection{GRB 060109}

At 16:54:41 UT on 2006 January 9, Swift-BAT triggered and located GRB 060109 (De Pasquale et al. 2006). The light curve of its prompt emission is shown in Fig. 1b. Its duration in 15$350 \mathrm{keV}$ is $T_{90}=116 \pm 3 \mathrm{~s}$, and the fluence in $15-150 \mathrm{keV}$ band is $(6.4 \pm 1.0) \times 10^{-7} \mathrm{erg} \mathrm{cm}^{-2}$ (Palmer et al. 2006). No redshift is measured for this event. If assuming a typical redshift of $z=1$, the isotropic-equivalent $\gamma$-ray energy release is $E_{\gamma, \text { iso }} \sim(3.74 \pm$ $0.53) \times 10^{51} \mathrm{erg}$. In the early X-ray afterglow, the light curve shows a rebrightening that begins in less than $1000 \mathrm{~s}$ and lasts for several thousand seconds.

Figure 3 illustrates our fit to the observed X-ray afterglow of GRB 060109 by using the the ring-shaped jet model. The parameters involved are given in Table 1 . In our modeling, we again omitted the observed steep decaying phase. We see that the theoretical light curve matches the observational data very well. The observed flux of the ring-shaped jet is about 10 times higher than that of a corresponding off-axis conical jet $\left(f_{\mathrm{r} / \mathrm{j}} \sim 10.4\right.$, see Table 1$)$. The ratio of $f_{\mathrm{o} / \mathrm{r}}$ is $\sim 48.5$. It rougly equals to $E_{\mathrm{K} \text {,iso }} / E_{\gamma \text {,iso }}$, which is about 18 as can be derived from Table 1. This proves that our modeling is self-consistent.

\subsection{GRB 070103}

At 20:46:39.41 UT on 2007 January 3, Swift-BAT triggered and located GRB 070103 (Sakamoto et al. 2007). The light curve 


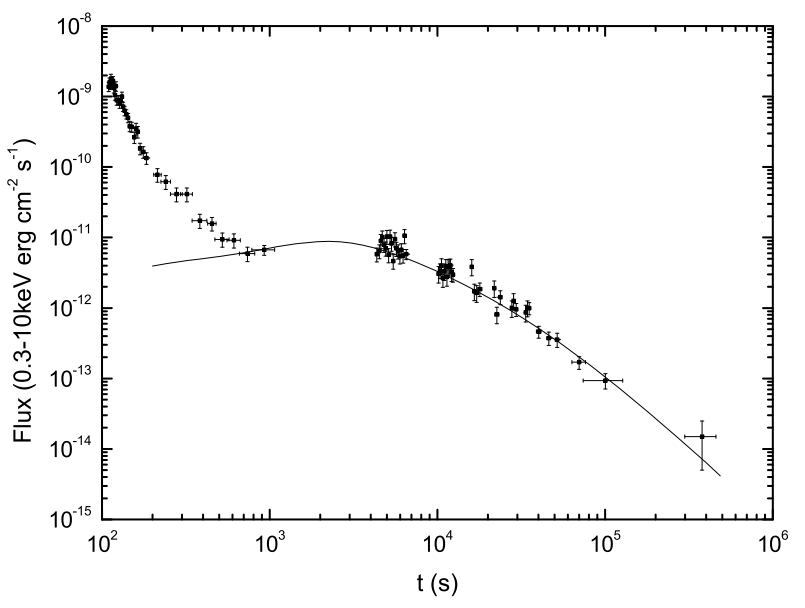

Fig. 3. Our best fit to the X-ray afterglow of GRB 060109 by using the ring-shaped jet model. The square data points are observed by Swift-XRT (see the Swift website).

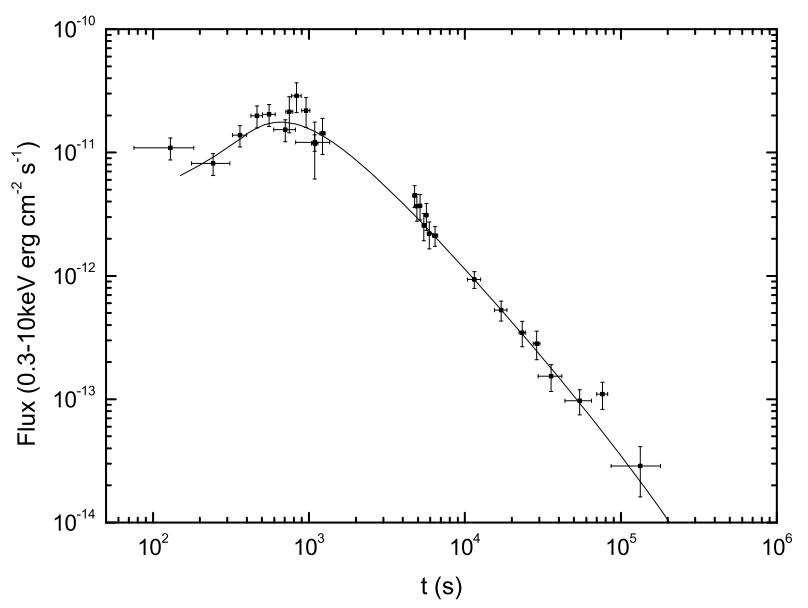

Fig. 4. Our best fit to the X-ray afterglow of GRB 070103 by using the ring-shaped jet model. The square data points are observed by Swift-XRT (see the Swift website).

of its prompt emission is shown in Fig. 1c. The duration in 15$350 \mathrm{keV}$ is $T_{90}=19 \pm 1 \mathrm{~s}$, and the fluence in $15-150 \mathrm{keV}$ is $(3.4 \pm 0.5) \times 10^{-7} \mathrm{erg} \mathrm{cm}^{-2}$ (Barbier et al. 2007). The isotropicequivalent $\gamma$-ray energy release is $E_{\gamma \text {,iso }} \sim(1.8 \pm 0.3) \times 10^{51} \mathrm{erg}$, assuming that the redshift is $z=1$. In the early X-ray afterglow, the light curve shows a rebrightening that begins at about $200 \mathrm{~s}$ and lasts for more than one thousand seconds.

In Fig. 4 we illustrate our best fit to the X-ray afterglow of GRB 070103 by using the ring-shaped jet model, with the involving parameters given in Table 1. Generally, the observed rebrightening and the overall light curve can be well explained. According to Table 1 , the value of $f_{\mathrm{r} / \mathrm{j}}$ is about 5.3 . Note that the parameter of $f_{\mathrm{o} / \mathrm{r}}$ is about 52.2, and the ratio of $E_{\mathrm{K}, \text { iso }} / E_{\gamma, \text { iso }}$ is about 22. Again these two values are roughly consistent with each other.

\subsection{GRB 070208}

GRB 070208 was triggered and located by Swift-BAT at 09:10:34 UT on 2007 February 8, (Sato et al. 2007). The light curve of its prompt emission is shown in Fig. 1d. The duration in $15-350 \mathrm{keV}$ is $T_{90}=48 \pm 2 \mathrm{~s}$ (Markwardt et al. 2007), and

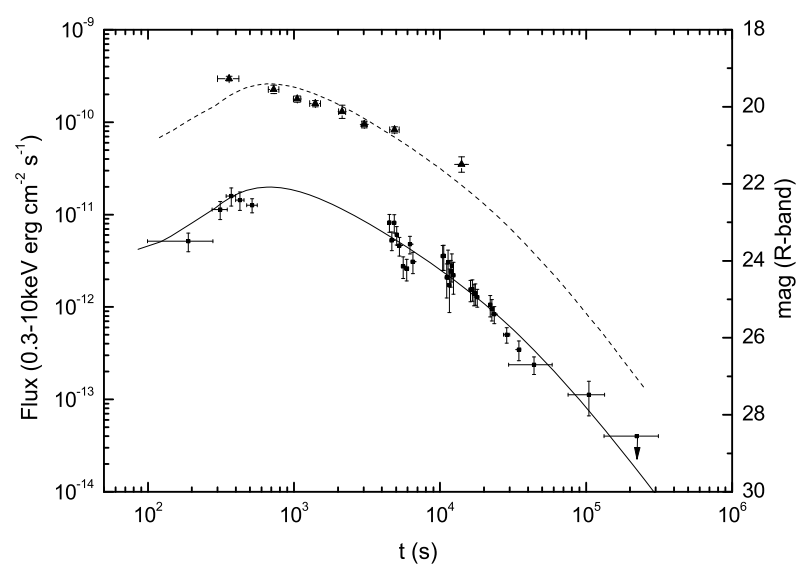

Fig. 5. Our best fit to the X-ray (solid line) and $R$-band optical (dashed line) afterglow light curves of GRB 070208 by using the ring-shaped jet model. The square data points are observed X-ray afterglow by Swift-XRT (see the Swift website) and the triangle data points are observed $R$-band afterglow (Cenko et al. 2009; Halpern \& Miraba 2007).

the fluence in $15-150 \mathrm{keV}$ is $(4.3 \pm 1.0) \times 10^{-7} \mathrm{erg} \mathrm{cm}^{-2}$. Lying at a redshift of $z=1.165$ (Cucchiara et al. 2007), the isotropicequivalent $\gamma$-ray energy release is $E_{\gamma \text {,iso }} \sim(3.32 \pm 0.77) \times 10^{51} \mathrm{erg}$. The X-ray afterglow light curve shows a rebrightening that begins at about $200 \mathrm{~s}$ and lasts for more than one thousand seconds (Conciatore et al. 2007).

Using the ring-shaped jet model, we have tried to fit the multiband afterglow of GRB 070208. The result is presented in Fig. 5, with the parameters given in Table 1 . The observed early rebrightening in the X-ray afterglow can be well reproduced. The optical afterglow can also be simultaneously explained. Table 1 indicates that the radiation intensity of the ring-shaped jet is about eight times more than that of a corresponding off-axis conical jet (i.e., $f_{\mathrm{r} / \mathrm{j}} \sim 7.7$ ). Note that the value of $f_{\mathrm{o} / \mathrm{r}}$ is about 61 . It again agrees well with the ratio of $E_{\mathrm{K}, \text { iso }} / E_{\gamma, \text { iso }}$, which is about 24 in our modeling.

\section{Conclusion and discussion}

It is possible that GRBs may be produced by ring-shaped jets. Interestingly, a few recent hydrodynamical and magnetohydrodymical simulations also give some support to this idea (Aloy \& Rezzolla 2006; Mizuno et al. 2008). A notable feature of the afterglow of a ring-shaped jet is that a rebrightening can be observed in the early afterglow stage, assuming that the line of sight is within the central hollow cone. In this study, we have clearly shown that the early rebrightenings observed in a few GRBs, such as GRBs 051016B, 060109, 070103, and 070208, can be well explained by the ring-shaped jet model. For GRBs 060109 and 070103 , of which only X-ray afterglow data are available, the fit result is very good. For GRBs 051016B and 070208, of which both the X-ray and optical afterglow data are available, the interpretation is also satisfactory. We propose that these GRBs with long-lasting rebrightening in the early afterglow are probably produced by ring-shaped jets.

Compared with a normal off-axis conical jet model, the advantage of our ring-shaped jet model is obvious. For a normal conical jet, when the observer is off-axis, the observed brightness will be notably reduced (as compared to the on-axis case). A normal conical-jet model then faces the problem of explaining the intensive $\gamma$-ray emission that is observed in the prompt GRB phase. On the other hand a ring-shaped jet can bring the 
complete material of the whole ring to contribute to the emission on the line of sight. The observed intensity can then be significantly enhanced compared to that of an off-axis conical jet. Indeed, for the four GRBs studied here, the amplification factor $\left(f_{\mathrm{r} / \mathrm{j}}\right)$ is generally $5-10$, compared with the corresponding offaxis conical jet.

From Fig. 1, we notice that all four GRBs show a similar 2 -pulse behavior in the prompt $\gamma$-ray light curve. This behavior is also observed in some other Swift GRBs (Butler et al. 2007). It is quite unclear whether this 2-pulse behavior is intrinsic to the mechanism that produces the ring-shaped jet or not. Huang et al. (2003) have argued that if the jet is produced by a rapid precessing process (with the precession period much shorter than the GRB duration), then the prompt $\gamma$-ray light curve could be highly variable and very complicated. It then seems that these four GRBs might not be caused by normal fast precession. However, a slow precession may still be possible. The structured jet mechanism by van Putten \& Gupta (2009) is of course another choice. Other possibilities include the evolution of an extreme Kerr Black Hole surrounded by a precessing disk (Lei et al. 2007), or the explanation of the second pulse as the tail emission from the first pulse.

From Table 1, we see that the initial angular radius of the inner edge of the ring is generally very small $\left(\theta_{\mathrm{c}} \sim 0.01-0.02\right)$. The width of the ring is also small ( $\Delta \theta \sim 0.02-0.08$ ). Taking typical parameter values of $\theta_{c}=0.015$ and $\Delta \theta=0.04$, we can give a rough estimate for the observed event rate of early rebrightenings. Among all Swift GRBs, the fraction of well-monitored afterglows with potential jet-breaks is around 60\% (Panaitescu 2007). For most of these jet candidates, the outflows might be normal conical jets, but it is quite likely that a small portion (here we take the ratio as $15 \%$, see Xu et al. 2008) of the jets are ring-shaped ones. For a ring-shaped jet with $\theta_{\mathrm{c}}=0.015$ and $\Delta \theta=0.04$, the possibility that our line of sight is within the central hollow cone (as compared with the possibility that the line of sight is just on the ring) is about 0.08 (calculated from $\left.\left(1-\cos \theta_{c}\right) /\left[\left(1-\cos \left(\theta_{c}+\Delta \theta\right)\right)-\left(1-\cos \theta_{c}\right)\right]\right)$. Finally, we estimate that the fraction of Swift events predicted by our model to display early rebrightening is $0.6 \times 0.15 \times 0.08 \sim 0.7 \%$. This number is consistent with the observed fraction of Swift events that show early X-ray rebrightening $(<1 \%)$.

The angular information may provide useful clues on the central engine of GRBs. In many progenitor models of GRBs, the central engine is a rapidly rotating compact star and the outflow is ejected along the magnetic axis. Our study then indicates that the inclination angle of the magnetic axis with respect to the rotating axis should be small $(\sim 0.02-0.06)$. In a few other progenitor models, the central engine is also a rapidly rotating compact star, but the outflow is ejected just along the rotating axis, then our results suggest that the processing (angular) radius of the rotating axis should be $\sim 0.02-0.06$.

In our framework, because the rebrightening is mainly a geometric effect, it should generally be achromatic, i.e. the rebrightening should appear simultaneously in all X-ray and optical bands. For the four GRBs studied here, the X-ray afterglow data are relatively prolific. But the optical afterglow data are generally so lacking that almost no rebrightening can be discerned in the optical light curves. Anyway, we have shown that our model is consistent with both the X-ray and optical observations. In the future, more examples with abundant multiband afterglow data will be available, and tighter constraints on the existence of ringshaped jet could be derived.
In our model, we assume the ring-shaped jet is uniform. But note that the actual structure of the GRB outflow may be very complicated. For example, it may be a two-component jet as predicted by some engine models (e.g. van Putten \& Levinson 2003). If these ingredients are included, then the afterglow behavior of ring-shaped jets will be correspondingly much more complicated.

Acknowledgements. We thank Fayin Wang and Yang Guo for helpful discussion. We also thank the anonymous referee for useful comments and suggestions that led to an overall improvement of this manuscript. This work was supported by the National Natural Science Foundation of China (Grant No. 10625313 and 11033002) and the National Basic Research Program of China (973 Program, Grant No. 2009CB824800).

\section{References}

Aloy, M. A., \& Rezzolla, L. 2006, ApJ, 640, L115

Barbier, L., Barthelmy, S., Cummings, J., et al. 2005, GCN, 4104

Barbier, L., Barthelmy, S. D., Cummings, J., et al. 2007, GCN, 5991

Butler, N., \& Kocevski, D. 2007, ApJ, 663, 407

Butler, N., Kocevski, D., Bloom, J. S., \& Curtis, J. L. 2007, ApJ, 671, 656

Cenko, S. B., Kelemen, J. F., Harrison, A., et al. 2009, ApJ, 693, 1484

Chen, Y. T., Wu, Y. L., Huang, K-Y., et al. 2005, GCN 4105

Chincarini, G., Moretti, A., Romano, P., et al. 2007, ApJ, 671, 1903

Conciatore, M. L., Stratta, G., Perri, M., Sato, G., \& Burrows, D. 2007, GCN, 6085

Cucchiara, A., Fox, D. B., Cenko, S. B., \& Price, P. A. 2007, GCN, 6083

Dai, Z. G., Huang, Y. F., \& Lu, T. 1999, ApJ, 520, 634

Dai, Z. G., \& Lu, T. 1998, A\&A, 333, L87

De Pasquale, M., Barthelmy, S., Beardmore, A., et al. 2006, GCN, 4455

de Ugarte Postigo, A., Fatkhullin, T. A., Jóhannesson, G., et al. 2007, A\&A, 462, L57

Fargion, D., \& Grossi, M. 2006, Chin. J. Astron. Astrophys. (ChJAA), 6, 342

Gehrels, N., Chincarini, G., Giommi, P., et al. 2004, ApJ, 611, 1005

Gehrels, N., Ramirez-Ruiz, E., \& Fox, D. B. 2009, ARA\&A, 47, 567

Granot, J. 2005, ApJ, 631, 1022

Halpern, J. P., \& Miraba, N. 2007, GCN 6082

Huang, Y. F., \& Cheng, K. S. 2003, MNRAS, 341, 263

Huang, Y. F., Dai, Z. G., \& Lu, T. 1999, MNRAS, 309, 513

Huang, Y. F., Gou, L. J., Dai, Z. G., \& Lu, T. 2000, ApJ, 543, 90

Huang, Y. F., Dai, Z. G., Lu, T., Cheng, K. S., \& Wu, X. F. 2003, ApJ, 594, 919

Huang, Y. F., Cheng, K. S., \& Gao, T. T. 2006, ApJ, 637, 873

Kocevski, D., \& Butler, N. 2008, ApJ, 680, 531

Kong, S. W., Wong, A. Y. L., Huang, Y. F., \& Cheng, K. S. 2010, MNRAS, 402, 409

Lei, W. H., Wang, D. X., Gong, B. P., \& Huang, C. Y. 2007, A\&A, 468, 563

Lipkin, Y. M., Ofek, E. O., Gal-Yam, A., et al. 2004, ApJ, 606, 381

Margutti, R., Doi, A., Tsuboi, M., et al. 2009, MNRAS, 402, 46

Markwardt, C., Barbier, L., Barthelmy, S. D., et al. 2007, GCN, 6081

Mizuno, Y., Hardee, P., Hartmann, D., et al. 2008, ApJ, 672, 72

Palmer, D., Ajello, M., Barbier, L., et al. 2006, GCN, 4476

Panaitescu, A. 2007, MNRAS, 380, 374

Parsons, A., Barthelmy, S., Beardmore, A., et al. 2005, GCN, 4103

Piro, L., Amati, L., Antonelli, L. A., et al. 1998, A\&A, 331, L41

Ramirez-Ruiz, E., Granot, J., Kouveliotou, C., et al. 2005, ApJ, 625, L91

Reichert, D. E., Lamb, D. Q., Fenimore E. E., et al. 2001, ApJ, 552, 57

Sakamoto, T., Barthelmy, S. D., Burrows, D. N., et al. 2007, GCN, 5988

Sato, G., Barthelmy, S. D., Campana, S., et al. 2007, GCN, 6074

Sharapov, D., Ibrahimov, M., Pozanenko, A., et al. 2005, GCN, 4185

Soderberg, A. M., \& Berger, E., Ofek, E. 2005, GCN, 4186

van Eerten, H. J., Leventis, K., Meliani, Z., Wijers, R. A. M. J., \& Keppens, R. 2010, MNRAS, 403, 300

van Putten, M. H. P. M., \& Levinson, A. 2003, ApJ, 584, 937

van Putten, M. H. P. M., \& Gupta, A. C. 2009, MNRAS, 394, 2238

Xu, M., Huang, Y. F., \& Kong, S. W. 2008, Chin. J. Astron. Astrophys. (ChJAA), 8,411

Yu, Y., \& Huang, Y. F. 2007, Chin. J. Astron. Astrophys. (ChJAA), 7, 669

Zhang, B. 2007, Chin. J. Astron. Astrophys. (ChJAA), 7, 1

Zhang, B., \& Mészáros, P. 2001, ApJ, 552, L35

Zou, Y. C., \& Dai, Z. G. 2006, Chin. J. Astron. Astrophys. (ChJAA), 6, 551 